\title{
Frequent discordance between clinical and musculoskeletal ultrasound examinations of foot disease in juvenile idiopathic arthritis observed in the multidisciplinary setting
}

\author{
Gordon J Hendry ${ }^{1 *}$, Martijn PM Steultjens ${ }^{2}$, Janet Gardner-Medwin ${ }^{3}$, Jim Woodburn², Debbie E Turner ${ }^{2}$ \\ From Australasian Podiatry Council Conference 2011 \\ Melbourne, Australia. 26-29 April 2011
}

\section{Background}

Ultrasound has shown promise for detection of sub-clinical disease in JIA. This may be particularly beneficial for the foot and ankle joints, which are difficult to examine in children. Early detection of sub-clinical foot disease permits earlier intervention which may improve outcome. The aim of this study was to evaluate agreement between clinical and ultrasound examinations of foot disease in JIA.

\section{Methods}

Thirty patients with JIA underwent clinical and US examination of 24 foot joints, 10 tendons and 6 periarticular soft tissues. Each site was examined independently by a rheumatologist and a podiatrist for synovitis, and tenderness/swelling. At the same sites the sonographer examined independently for effusion, synovial hypertrophy, power Doppler signal (PS), tenosynovitis, or abnormal tendon thickening. Agreement was estimated using Cohen's unweighted kappa $(\kappa)(>0.4=$ moderate agreement) with associated $95 \%$ confidence intervals.

\section{Results}

720 joints, 300 tendons and 180 soft tissue sites were assessed. Clinically detected synovitis, tenderness and swelling were recorded in $42(5.8 \%), 78(10.8 \%)$ and 73 (10.1\%) joints respectively. US-detected effusions, synovial hypertrophy and PS were recorded in $88(12.2 \%), 47(6.5 \%)$ and $12(1.7 \%)$ joints. Tenderness and swelling were recorded in 29 (9.7\%) and 16 (5.3\%)

\footnotetext{
* Correspondence: gordon.hendry@gcu.ac.uk

${ }^{1}$ School of Biomedical and Health Sciences, University of Western Sydney, Sydney, Locked Bag 1797, Australia

Full list of author information is available at the end of the article
}

tendons and 28 (15.6\%) and 9 (5\%) soft tissues. USdetected tenosynovitis and PS were detected in $7(2.3 \%)$ and $6(2 \%)$ tendons. Abnormal thickening of the plantar fascia origin and Achilles tendon insertion were detected at a frequency of $4 / 60(6.7 \%)$ and $1 / 60(1.7 \%)$, and $3 / 60$ (5\%) effusions were recorded at the retro-calcaneal bursa. Subclinical foot disease was discovered in 52 (7.2\%) joints, $5(1.6 \%)$ tendons and $4(2.2 \%)$ soft tissue sites. Agreement was consistently less than moderate $(\kappa<0.4)$ for each clinical and US interaction. There was moderate agreement between the rheumatologist and podiatrist for active synovitis versus joint swelling $(\kappa=0.52)$.

\section{Conclusions}

There is frequent discordance between clinical and US assessments of foot disease in JIA. Subclinical foot disease appears common; however clinical examination also detected features of active disease in structures that were recorded as normal on US. These findings suggest US may be a useful tool to aid clinical examination of the foot in JIA patients.

\section{Author details \\ ${ }^{1}$ School of Biomedical and Health Sciences, University of Western Sydney, Sydney, Locked Bag 1797, Australia. ${ }^{2}$ Glasgow Caledonian University, Glasgow, G4 0BA, UK. ${ }^{3}$ University of Glasgow, Glasgow, G12 8QQ, UK.}

Published: 20 May 2011

doi:10.1186/1757-1146-4-S1-019

Cite this article as: Hendry et al:: Frequent discordance between clinical and musculoskeletal ultrasound examinations of foot disease in juvenile idiopathic arthritis observed in the multidisciplinary setting. Journal of Foot and Ankle Research 2011 4(Suppl 1):O19. 